Pub. Mat. UAB

no 11, jarç 1979

\title{
ESPACIOS DE SUCESIONES
}

por

Manuel Tort

Sa llame especto de eucesiones a todo subespecio vectorial del espacio $w$ de todes les strestones de nimeros reeles o conplejas. La taoria da los aspactos de suceslones fus deserrollada principalmente por KBthe $y$ Togplitz $[12]$ en 1934 y posteriormente por Kothe [9] y [10] , si bien se encuentran precedentes en trabajas anteriores. Su desarrolio esta 11gado, al orinctpio, al de las teorfas de log especios de Hilbert $y$ de los espectas nomados. Más adelanta, el deserrollo de la tearla de los espaciog localnente convexos supuso nuevos avances en el estudio de los espotios de suceB1ones.

Exponamos, a continuation, algunas de las fdaas y de los trebejos que llevaron a la consideractón de los espacios de sucesiones, a La defintción y egtudio de los especiog nomados $y$, en general, de los espacios vactorialas topologicos.

En el estudio de las ecuecionas 1ntegrales Iingeles del tipo

$$
u(x)+\int_{a}^{b} K(x, y) w(y) d y=f(k)
$$

para la función incógnita $u$, on 1903, I. Fredhoin considero la enalogfa entra Ástas y los ststamas Innales

$$
\sum_{q=1}^{n}\left(\delta_{p+q}+\frac{1}{n} a_{p+q}\right) x_{q}=b_{p}(p+p<n)
$$

para obtener la saluctón de la ecusción Integral como cociente de dos exprestonas, sigutando el método de Cremer. Esta 1déa sa basa an el matodo de los coeflefentes fndeterainados, consistente an haller une functón tnefgnite, qua se supone deserrollable an serle 
$\sum_{n=1}^{0} c_{n}$ (donde les $\varphi_{n}$ san punctones conocides), calculanda los cooplolertos $c_{n}$. Esta método habfa llevedo a la consideración de Blstemes dineales con infinftes incógntis

$$
\sum_{j=1}^{\infty} a_{i, j} x_{j}=b_{i}, \quad i=1,2, \ldots .
$$

En 1906, H1Ibert, ut1ilzando el método da los cosfictentes 1ndeteminedos, muestra que la salución de la ectación integral

$$
u(x)+\int_{d}^{b} K(x, y) u(y) \alpha y \alpha f(x)
$$

es equivalente a la del sisteme de Infinites ecuaciones lineales

$$
x_{p}+\sum_{q=1}^{\infty} x_{p+q} x_{q}=b_{p}, p=4,2, \ldots .
$$

donde las incognitas son los coeflelentes de Fourter

$$
x_{p} * \int_{j}^{b} u(t) w_{p}(t) d t
$$

de la función incognita u respecto a un sisteme ortanomel campleto $\left(\omega_{n}\right)$

$\left(\operatorname{con} b_{p}=\int_{a}^{b} f(t) \omega_{p}(t) d t . \quad y k_{p q}=\int_{0}^{b} \int_{a}^{b} K(s, t) \omega_{p}(s) \omega_{q}(t) d s d t\right)$. Lag soluctanes que coneldera son aquallas para las cuales $\sum_{n=1}^{\infty} z^{*}<+\infty$ Es in estos trebajog donda eperece le fuea del espacio de las sucesiones de cuedrado sumeble, si bien no se ha introducte explicttarante. Este espacto se presenta camo un paso el limite pertir de los espectos eusildecs de dimensión flnita. Hilbert intraduce en este espacio dos tipos de convergencta qua son las que so han 1lsmedo postertornento comergencle débil y convergencle fuerte.

En 1908, F. Rleaz Introduce los espactos $L^{P}(I)$ de las functonaa te potencia $p-6$ sina integrable $(1<p<\infty$, estudio al ate le algue, tres axłog más tarde, un traba to enálago donde se intraducen lós appelos $L^{P}(N)$ de las sucestonas de potencta o-ésime sumable F se astudia la dualidad entra estos aspacias. Sabre este cuestión cábe of tar un reaultado orevio demostrado por Landau en 1907 nue 
establece que la convergencia de le serte $\sum_{n=2}^{\infty} u_{n} x_{n}$, pare toda 'edicesión $\left(x_{n}\right) \in L^{P}(N)$, implica que la sucesión $\left(u_{n}\right)$ pertangee a $L^{q}(N)\left(\operatorname{con} \frac{1}{p}+\frac{1}{4} * 1\right)$.

La definición genaral de los espaclos normedos fue dade par 5. Banach, H. Mahn y E. Melly, aunque śste ultimo considera sólamente espacios de sucesiones de núneros realas o corplejos.

En 1921, Heliy intraduce una noctón de narma en: los espacios de sucesiones que generalize el funclonel de Minkowsk1 de un cuerpo convexo en los espacios de dimenglón finita. Si $E$ es un espac10 de sucesiones dotado de une narma, establece una dualidad entre $E$ y el espacio $E^{\prime}$ formado por las sucesiones $u=\left(u_{n}\right)$ tales que, para tode $x *\left(x_{n}\right) \in E$, la serie $\sum_{n=1}^{\infty} u_{n} x_{n}$ sea canvergante. Define une noma on $E^{*}$ por Hul= $\left.\sup _{x \neq 0} \mid\langle u, x\rangle\right\} / N \times H$. A partir de estoo conceptos, la resolución de un slstema en $E$

$$
\sum_{j=1}^{\infty} a_{i j} x_{j}=b_{i}, \quad i=1,2, \ldots,
$$

donde los $u_{i}=\left(a_{j}\right) \in E$; se reduce a resolver las siguientea cuestianes: 1) Heller una forme lineal continua $L$ sobre $E^{*}$ tal que, para todo $1 \in N$, se verifique $\left.L\left(u_{i}\right)-b_{i}, 2\right)$ Determinar si existe alguín $x \in E$ tal aus, para tado $u \in E^{*}$, se cumple $L(u)=\langle u, x\rangle$. Para este ditimo problema no slempro existo solución. como abgerve Helly, aun cuando $L$ exista, $y$ se limita a dar condicianes suficientes en cesos perticulares.

En 1927, gl procedimiento de Minkowski-Helly es ut1llzedo por H. Hahn en un espacio normado cualquiera para defintr an el dual una estructura de espacio normado completa. Estos resultados son. obtenidas dos años más terde, de un modo independiente, por $\mathrm{S}$. Benach. Estos rasultados preperan la defin1c16́n de las espacios locelmante convexos, dade por J. von Neumann en 1935.

Finalmente cabe destacar la teoria de los especios de sucesiones inlclede por Kothe y Toeplitz on 1934 y deserrolleda on años sucesivas por Kothe. Esta he ut1lizado para su desarrollo 103 avam- 
Goo on la taoria de las espaclos locelmante convexos y ha servido Se codolo a Sater Ultima proporcionanda diversos cantraejemplas.

Exponenos, a continueción, las definiciones y principalas resultadas de esta teorla (KBthe [11] 530 ).

814 es un conjunto te sucesianes, al conjunto formado por tadas las $y=\left(y_{n}\right) \in \omega$, con $\left|y_{n}\right| \leq\left|x_{n}\right|$, pere alguna $x=\left(x_{n}\right) \in M$, 8* Ilane la envaltura narmal de M. Un conjunto de sucesianas se liene namel o bolido si colnetide con su envoltura nomel.

Se repregenta por $\rho$ ol espacto de sucesiones formado por la que tienen únicemente un número finito de términos distintos de cord. Pexa cada $n \in N$, se represente por $e^{n}$ la sucesión $\left(x_{k}\right)$, donde $x_{4}=1$ si $k=n \quad y \quad x_{k}=0$ si $k$ \& $n$. Las sucesiones $a^{n}$ Pormen base del espacio $\varphi$. See $x=\left(x_{n}\right)$ un elemento de $\omega$. Para cada a $\in N$ se define $x^{m}=\left(x_{n}^{m}\right)$ por $x_{n}^{m}=x_{n} \quad 5 \pm$ n $\leq m y$ $x_{n}^{m}=0$ st $n>m$. Las sucestones $x^{m}$ se llaman las secclones da 4 $y$ partenecen al especio $\varphi$.

A cada espacio de suceglones $\lambda$ se le asigne otro, $\lambda^{\not}$ su a-dual, formada por todas las bucesiones w a $\left(u_{n}\right)$ teles que la serte

$$
\sum_{n=1}^{\infty} u_{n} x_{n}
$$

sea absolutamente convergente, para tode $x=\left(x_{n}\right) \in \lambda$.

$\lambda^{x}$ eg un especio de sucesiones normal que contiene $\varphi$. Un espacio de aucestones se llama perfecto o de Kathe st $\lambda_{a} \lambda^{x x}$. $51 \lambda$ es'un aspacio de sucesionas, $\lambda^{x x}$ eg el minimo especio perPecto que confiene $\lambda$. Un espaclo perfecto es normel y contiens $\}$. Los espacios $\varphi$ y w son perfectus $y$ of-dueles el uno del otro. Los espectos $1^{\prime}$ (espacio de las sucesiones $\left(x_{n}\right)$ tales nue Ia serie $\sum_{n=1}^{\infty} x_{n}$ es absolutamente convergente) y $1^{\infty}$ (espacta de les sucesiones ecatedes) son perfectos y $\alpha$-duales el una del otro. Para todo númaro real p > l, los espectos lí (espacio de las su- 
casiones tales que la serte $\sum_{n=1}^{\infty} \mid x_{n} t^{r}$ es canvergente) son perfactar $y$ se tiene

$$
\left(l^{p}\right)^{x}=l^{1}, \text { con } \frac{t}{p}+\frac{1}{q}=L
$$

$51 \lambda$ as un espacto de sticestones que contiene $y, \lambda y$ su $\alpha$-dual $\lambda^{\alpha}$ forman sisteme dual con le forme bilingal

$$
\langle u x\rangle=\sum_{n=1}^{\infty} u_{n} x_{n}
$$

pera $u=f\left(u_{n}\right)=\lambda^{x} y x=\left(x_{n}\right) \in \lambda$. Las tooologfas débtl, de Meckey $y$ fuerta on un especio $\lambda$ aje contenga $y$ se considerarán stempre relativas al ststema dual $\left(\lambda, \lambda^{x}\right)$.

Une sucesion $\left(x_{n}\right)$ se Ilane positiva si $x_{n} \geqslant 0$, pere toda $n \in N . S 1 u=\left(u_{n}\right) \in \omega$, se rapresenta por $\lambda_{n}$ el $\alpha$-dual de la envoltura ineal de 4. S1 $\lambda$ es un espacto perfecto, $\lambda$ ednita le reprasentactón

$$
\lambda=\bigcap_{\substack{u \in i^{*} \\ s<}} \lambda_{u}
$$

pare los elemantos $u=\left(u_{n}\right)$ pasitivos de $\lambda^{x}$.

En el conjunto de las sucegiones posttives se define Ia releción de orden: $a \leqslant b$ si $b-a$ eg posteiva. 51 a $\leq b$, se tiena $\lambda_{3}>\lambda_{b}$. Ell especio $\lambda$ gs Isconorfo el IImite proyectivo do las espacios $\lambda_{H}$, pare las alementos positivos de $\lambda^{\times}$, con les aplicaciones inclusión entre estos espacios.

See $\lambda$ un espacto te sucesiones que contiene $\varphi$. La topologla definlda por el sistema de seminormas

$$
P_{n}(x)=\sum_{n+1}^{\infty} t m_{n} x_{n} t
$$

pare $u=\left(u_{n}\right)+\lambda^{x}$. se llame le topologle nomel en $\lambda$. La topologla normal es la de Ia convargenela uniforme abre las amaltu-" ras nomales de los elementos de $\lambda^{*}$. Pugsto que astas conjuntos son absolutamente canvexas y dobilmente compactos, la topologfa normal es competible con el sistema dual $\left(\lambda, \lambda^{x}\right)$.

En el especto $2^{\prime}$ cotncite la topalogia normel con la def1- 
nita por la normi $|t| x\left|=\sum_{n=1}^{\infty}\right| x_{n} \mid$. Los especios $\lambda_{k}$ san de Fréchet cen 2 e copologla nomal.

60 A un espacto perfecto. $\lambda$ con la topologfa normel as topologlcamente 1somorfo el Ifmita proyectivo de los especios $\lambda_{w}$, dotados de Ia tapologia normal, para los elementas positivos de $\lambda^{x}$, con Ias aplicscianes tncluatón entre estas espacios. Por lo tanto as topologlcantente 1sonorfo a un lintte proyecttvo de espacios da Frochet. De esta representeción de los espacios perfectos se deduce una caracterizeción topologlea de astos espactos: un especio da sucestones que contiene $\varphi$ ss perfecto a1 y s6lo 31 es dabtimente completa por sucestones y st y s610 51 es complato con la topologla nomal.

En un especto que contenge $\varphi$ las secclones de una sucesión convergen hecla elia por le topologla débil y por le nomel. St ademág el espacto es normel, las sacclonas converyer por la topologla de Mackey. Un espaclo parfecto es tonglado con la topologle de Mackey st y 5610 st las secclores de une sucestón convergen por la topologle fuerte.

Un resultedo interesente relativo a Ios subconfuntos compectos do los espacios perfuctas establece our en un especio perfecto con une topologla más fine que la débll colnetden los conjuntos compectos, los numerablemente compactos y los conpectos par sucasiones. En un espacio perfecto colneiden los conjuntos debilmante compactos con Ias compectos pere la topologla normal y las sucesiones debilmente convergentes con les convergantes para is topologle nomal.

S1 $\lambda$ es un esperto perfecto, la topologie de Mackay en $\lambda^{x}$ es la poler de ia nomel en $\lambda$ (topolagla de le convergancte precompacte) y la polar de le de Meckey en $\lambda^{x}$ es le topologia en $\lambda$ locelnante convexa más fine que tiene los mismos conjuntos compactos y las migras sucesiones convergentes que la dobll. Es compatible con el b1stena turel $\left(\lambda, \lambda^{x}\right)$. 


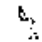

Consioeraremos, a continugción, une clerta clase de espacios perfectos: los espacios escelonedas y co-escalanadog. Estos espacios fueran Introducidos por KBtie [9] en 1948. Se sstablece un mátodo canstructivo de aspacios perfectos a pertir de une sucesion de sucesiones. Los espacios escalonados son espacios de fréchet con 10 topolog la normal.

Dade une sucesión de stucesiones positivas $a^{k}$, tal que $a^{k} \leq$ $a^{2} \leq \ldots . \leq a^{k} \leq \ldots$, se dice que $a^{k}$ es un sigtema creclente de escalones, el cial se llama completo si pere cada $n \in N$ exista un $k \in N$ tal qure $\boldsymbol{B}_{n}^{m}+0$.

Dado un sistema creciente $y$ campleta de escalonas a $a^{k}$, $0 s$ espacios

$$
\lambda=\bigcap_{k=1}^{\infty} \lambda_{a^{k}} \quad y \quad \mu=\bigcup_{k=1}^{\infty}\left(\lambda_{a^{N}}\right)^{k}
$$

se llamen las espacios escelonedo y co-escalanado, respectivamente, correspondientes el sisteme de escelones ak . Estos especios son Perfectos $y \quad \alpha$-duales el uno dal otro.

Más adelante, este concepto as ganeralizado por Dleudonné y Gomes [5], definienda los espectos escelonados y comescalonados de arden $p$ (donde $p$ es un número real mayor o igual que uno) de rado que pere $p=1$ se tienen Ios espacios definidos por Kothe.

S1 $p$ es un número real mayor o 1gual que una, se define el espacto $\lambda_{u}^{p}$ como el farmado por las suessianes $\left(x_{n}\right)$ tales awe la ser1e

$$
\sum_{n=1}^{\infty}\left|u_{n}\right|\left|x_{n}\right|^{p}
$$

es convergente. El espacio $\lambda_{\mu}^{p}$ calncide con $\lambda_{u}$ en el caso $p=1$. A partir de los especios $\lambda_{\mu}^{p}$, se defiten los especios escelonedos y co-escalonados de orden $p$ de un modo análogo a la definición de los enteriores especios.

Deda un sistema crecienta y completo de escalones $a^{k}$ y un nómero real p mayor a Igual que uno, los espacios

$$
\lambda=\bigcap_{n=1}^{\infty} \lambda_{a^{k}}^{p} \quad y \quad \mu=\bigcup_{k=1}^{\infty}\left(\lambda_{\mathbf{a}^{4}}^{p}\right)^{k}
$$


se 1keman las espactos escalonado y co-escalonado de arden $D$, respectivamente, correspondientes al sisteme de escalones $a^{k}$. Estos especios son perfectos $y$-duales al uno del otro, Para $p>1$, el espacio escelanado de orden $p$ es un espacio de Fréchet reflexivo con la topalogia de Mackey.

Une nueva generalizecion fue establecida por Oublngky [6] en 1967, caracterlzando los espec10s parfactos oue son de Fréchet con la topologie fuerte.

Se llane escelón a todo espacio perfecto $\lambda$ tal que $\lambda^{\prime} \subset \lambda<1^{*}$ y sea de Barach con la topologfe fuerte. Sea $\left(\lambda_{n}\right)$ una sucagión de escalones $y\left(a^{k}\right)$ ung sucestón de sucesianes tales que

1) $0<a_{i}^{k}<a_{i}^{k+1}$, para todo $1, k \in N$, 2) $1 / a^{k+1} \lambda_{k+1}=1 / a^{k} \lambda_{k}$, pare todo $k \in N \cdot \operatorname{Los}$ espec1os

$$
\lambda=\bigcap_{k=1}^{\infty} \frac{1}{a^{k}} \lambda_{k} \quad y \quad \mu=\bigcup_{k=1}^{\infty} \theta^{*} \lambda_{k}^{x}
$$

ee Ilamen los especios escelonado $y$ co-escelonedo, respectivamente, sobre el sistema de escalones $\left(a^{k}, \lambda_{h}\right)$. Estos espacios son perfectos y d-duales al uno del atro. El espacio escelonacto es de Fróchet con la topologla fuerte. A partir de éstos se caracterizan todos Ios mspacios perfectos que son de Frechet con le topologia fuerte, s1 blen estos ultimas no coinciden con los espacios escalonedos. No abstente, como geñela Dubinsky en su trabejo, $Y$. Komure ha observado que s1 se debilisa la hipbtesis sabre el sistema de escelones, suponiendo $0 \leq \theta_{i}^{k} \leq a_{i}^{k+t}$, la conetrucción algue sienda válida y los espacios escalonados abtenidos de este modo cainciden con los espaclos perfectos que son de Fréchat con la topologla fuerte.

Le teorle de los especios de sucestones he servido coma modelo a la teorfe de los espacios vectariales topoldgicos y se ha utilizado pera dar contraejemplos en esta teorfa. Citaremos, a continuación, elgunas de ellos. 
Mediante un Espacio escelonedo se da un ejenplo de un espacia que es de Frechet $y$ de Montel en el que existe un cociente cuse no es espacio de Montel. Al mismo tiempo, este ejemplo demuegtra ave un. coclente de un especio de fréchet reflexivo no es neceserianente refiexivo. El $\alpha$-dual de este espacio, con la topalogia fuerte, praporcione un ejemplo de un espacio DF en el que existo un subespacto cerrado que no es DF. Tamblén, medianto un especto escelanada, se da un ejemplo de un espacio de Fréchet no distinguido (Kathe [9] y Grotinandieck [7]). Este Ultimo ejemplo fua utilizeda más tarda por Anemiya [1] para tar un ejamplo de un espacio DF bornológlco cuyo bidual fuerte no ss bornolágico.

En 1963, T, Y. Komura [B], utilizzando la hlobtesls del corstInuo, contesten negativamente a dos cuestiones propuestas por Kothe y por Dieudonné. Katha se pregunteba si todo espacto perfecto tonelado era bornológlca y Dieudonná si el completada de un espacio bornalogico ere bornológlco. A partir de un espacio de KBtha $A$. definen el especio $\lambda_{0}$ camo la edherencta de $\varphi$ can le topología bornológlce asoctade a la fuerte. $\lambda_{0}$ es un especio de sucesiones bornológico can Ia topología Inducide aor la fuerte an $\lambda$. Dan un ofemplo de un especio perfecto $\lambda$ que con la topalogia fuerte es tonelada $y$ no bornológico. Este espacio es el completedo del espacio bornológico $\lambda_{0}$. con lo que quadan cerradas las dos cuestiones.

La teorfa da Käthe sobre las especios de sucestones ha stdo gerneralizada en varios sentidos: a espacios vectorleles con un flgebra de Goole de proyecciones por Cooper [3], a especios de sucestones con valores en un espacio localmente canvexo por Plitach [14], a espacios de funciones localmente integrables oor 01eutonnó $[4] \mathrm{y}$, en el ceso reel, a espectos perclelmente ordenedog por Luxemburg y Zaanen [13]. Tembién se han considerado otros tioas de duelidad, como la $\beta$-dualidad. S1 $\lambda$ es un especio de sucestones se dafine $\lambda^{\circ}$; su $P$-cual, como el espacia de las sucastones $\left(u_{*}\right) \in \omega$ tales que, 
pare toda $\left(x_{n}\right)$ a $\lambda$, Ia serie $\sum_{n=1}^{\infty} \omega_{n} x_{n}$ seg convergente. El $\beta$ ous de un espacio contiene el $\alpha$-dual y si el espacio es nomal apbos duales colnciden. Un espacio de sucesiones $\lambda$, que contiene $\varphi$, $y$ su $\beta$-duel forman sistema duel can le forme bilineal $\left\langle u_{1} x\right\rangle=\sum_{n=1}^{\infty} u_{n} x_{n}$, para $u=\left(u_{n}\right) \& \lambda^{\beta}$ y $x=\left(x_{n}\right) \in \lambda$.

Degcribiremas brevemente la generalización de Dieudonné a espacios de funciones localmente integrables. Los espacios de sucasiones se presentan al canstderar $N$ con la topologie y la med16a discretes.

6ea E un especio topológico locelmante compacto numereble al Infinito con una medida de Aadon positiva. Se rapresente por $\Omega$ al espacto de las clasas de funciongs localmente integrables en $E$. $51 r$ es une parte de $\Omega$, el conjunto $\wedge$ formedo por las funciones $f$ a tales que, pera toda $g \in \vec{r}$, fg sea Integrable es un subesfacio vectorial de $\Omega$; se dice que $\Lambda$ es un espacio de Kethe y $\Gamma$ un conjunto de definición de $\Lambda$. Se define $\Lambda^{x}$ como el especio de Kothe definido sobre $\Lambda$. $\Lambda$ y $\Lambda^{*}$ contienen el espec1o $\oint$ (especio de las clases de funciones medibles y acotades con soporte compacto) y forman sistema dual con la forma bilineal

$$
\langle p \cdot g\rangle=\int_{\Omega} f g \alpha \mu
$$

Se estudian topologles reletivas a esta dual1dad y 58 generalizan resultados establecidos pere especios de sucesiones.

\section{BIBLIOGAAFIA}

[1] ANEAIYA, I. "Some examplas of (F)- and (DF)-spaces" Proc. Japen Acad.33, 169-121 (1957).

[2] BoluaAkI, N. "Elementos de histaria de las matemátices" Alienze Editor1al $\{1972\}$.

[3] COOPEA, J.L.日. "Coordinated I1near spaces", Proc. Landon Msth. 
Soc., III. ser. 3, 305-327 (1953).

[a] DIEUOONNE, J. "Sur les espaces de Kätho". J. Analyse Math. I, 81-115 (1951).

[5] DIEUDONNE, J. y GOAES, A.P. "Sur certeins espaces vectoriels topologiques". C.R. Acad. Sc1. Parts 230, 1129-1130 (1950).

[6] OL8INSKY, E. "Perfact Fróchet speces". Wath. Ann. 174, 186-194 (1967).

[7] GROTHENDIECK, A. "Sur les aspaces $(F)$ et (DF)". Suma Bragll. Math. 3, 57-123 (1954).

[B] KOAUAA, T. e Y. "Sur las especes parfaits de suites et leurs yénéralisations". J. Math. Goc. Japan 15, 319-338 (1963).

[9] KÖTHE, B. "Dio Stufenräume, elne elnfache Klesese linearer vollkomnener Aäumg". Mgth. Z. 51, 317-345 (1948).

[10] КӧTHE, G. "Neubsgründung der Theorle der vollkommenen aẽume". Math. Nachr. 4, 70-e0 (1951).

[11] KÖTHE, G. NTopologleal vector speces I'. Oxe Grundlheren der Mathemat1schen W1ssenschaften B.159 Springer-Verlag (1969). [12] KÖTHE, 6. y TOEPLITZ, 0. "Lineare BBume mit unendlichen vielen Koordinaten und ainge unandlichar Matrizen". J. raing angaw. Math. $17,193-226(1934)$.

[13] LUXEMBUAG, W.A.J. y ZAANEN, A.C. "Nates on Benach functian spaceg". VIII-XIII. Naderl. Aked. Wetensch. Indag. Wath. 25, 104-119, 360-376, 493-543 (1964).

[14] PIETSCH, A. "Verallgemelnerte vollkommene Folgenrtume" . Sohriftenreihe d. Institute d. Math. Deutsche Akad. Wise. Helft 12, Akadem1e-Vorlag Berlin (1962). 